\title{
ASSOCIAÇÃO DA DISLEXIA DO DESENVOLVIMENTO COM COMORBIDADE EMOCIONAL: UM ESTUDO DE CASO
}

\author{
Association between developmental dyslexia \\ and emotional co-morbidity: case report
}

\author{
Ricardo Franco de Lima (1), Cíntia Alves Salgado ${ }^{(2)}$, Sylvia Maria Ciasca (3)
}

\section{RESUMO}

Objetivo: analisar características em um caso de dislexia com depressão infantil. Método: descrição dos resultados da avaliação interdisciplinar em um caso com dislexia e depressão. Participou deste estudo uma criança, do gênero masculino, com 10 anos e 7 meses, cursando o $4^{\circ}$ ano do Ensino Fundamental em uma escola pública. Resultados: os resultados deste estudo evidenciaram alterações não somente na linguagem, em que há dificuldades no processamento fonológico da informação, como também na atenção e funções executivas e quadro comórbido de depressão infantil. Conclusão: o estudo revelou a necessidade da avaliação de uma equipe interdisciplinar para um diagnóstico preciso e, conseqüentemente uma melhor intervenção em crianças com dislexia e suas comorbidades.

DESCRITORES: Dislexia; Avaliação; Depressão; Comorbidade

\section{INTRODUÇÃO}

De acordo com o DSM-IV-TR 1 a Dislexia do Desenvolvimento é definida como um transtorno específico de aprendizagem, caracterizada por um desempenho escolar na leitura/escrita inferior ao esperado para a idade cronológica, escolaridade e ao nível cognitivo/intelectual do indivíduo.

Do ponto de vista neurológico, a dislexia é considerada uma disfunção do Sistema Nervoso Central, que compromete a aquisição e o desenvolvimento das habilidades escolares, tendo como critérios de exclusão o rebaixamento intelectual, déficits sensoriais (visual, auditivo), déficits motores significativos, com condições supostamente adequadas de aprendizagem e ausência de problemas psicossociais².

(1) Neuropsicólogo; Laboratório de Pesquisa em Dificuldades, Distúrbios de Aprendizagem e Transtornos da Atenção (DISAPRE), Campinas/SP; Mestrando em Ciências Médicas na Universidade Estadual de Campinas.

(2) Fonoaudióloga; Laboratório de Pesquisa em Dificuldades, Distúrbios de Aprendizagem e Transtornos da Atenção (DISAPRE), Campinas/SP; Doutoranda em Ciências Médicas na Universidade Estadual de Campinas.

(3) Neuropsicóloga; Livre Docente em Neurologia Infantil da Universidade Estadual de Campinas.

Conflito de interesses: inexistente
A dislexia pode ser dividida nos tipos: a) disfonética ou fonológica (auditiva), caracterizada por dificuldades na leitura oral de palavras pouco familiares, na conversão grafema-fonema e possível disfunção no lobo temporal; b) diseidética ou superficial (visual), caracterizada por dificuldade no processamento visual, na qual a criança lê por meio de um processo elaborado de análise e síntese fonética e disfunção no lobo occipital; c) mista, caracteriza por dificuldades dos dois tipos anteriores, sendo associada à disfunção dos lobos pré-frontal, occipital e temporal ${ }^{3}$.

Outra classificação divide a dislexia em outros subtipos, em função do desenvolvimento da leitura e da linguagem, reconhecimento da palavra e compreensão de leitura: leitores e compreendedores pobres, leitores médios e bons leitores ${ }^{4}$.

Indivíduos com dislexia apresentam déficits específicos nas funções neuropsicológicas, tais como no processamento visual e auditivo, no sistema fonológico da linguagem ${ }^{5-8}$, na atenção ${ }^{9} \mathrm{e}$ nas funções executivas ${ }^{10}$.

Estudos de caso com crianças com dislexia do tipo mista, evidenciaram déficits em provas específicas de leitura/escrita, consciência fonológica, maior frequência de erros na escrita sob ditado de palavras irregulares de baixa frequência, palavras 
inventadas irregulares e com regra, trocas surdassonoras na escrita, em memória de curto prazo e de trabalho, organização percepto-motora, falta de concentração, sinais neurológicos menores (soft signs) além de indicaram a importância da realização de avaliação interdisciplinar para o diagnóstico 2,8 .

Observa-se que crianças com distúrbios de aprendizagem podem ter alto risco para o desenvolvimento de transtornos mentais, uma vez que tendem a ter baixo auto-conceito, alto locus de controle externo, serem menos aceitas socialmente e mais ansiosas que seus pares sem dificuldades de aprendizagem ${ }^{11}$. Um estudo ${ }^{12}$ indicou que indivíduos com dificuldades na leitura apresentam maiores níveis de depressão, traços de ansiedade e queixas somáticas, ou seja, de comportamentos internalizantes, quando comparados a leitores fluentes.

Outro trabalho ${ }^{13}$ mostrou haver relação entre as dificuldades severas e persistentes de leitura e o aumento no risco para o humor deprimido em meninos de 7 a 10 anos de idade.

Em trabalho mais recente ${ }^{14}$ foi obtida taxa de prevalência de $9,09 \%$ de sintomas depressivos em crianças com dificuldades de aprendizagem avaliadas com o CDI. Além disso, quanto maior a idade cronológica, maior foi o número de relatos de ideação suicida passiva e preocupação.

Um estudo de revisão e meta-análise ${ }^{11}$ identificou que os estudantes com distúrbios de aprendizagem exibem escores maiores em testes de depressão quando comparados com indivíduos sem dificuldades. Os autores destacam que os estudos revisados apresentam dados de sintomatologia e não de depressão clínica para um transtorno depressivo maior.

Apesar do aumento no número de estudos internacionais que descrevem os déficits encontrados na criança com dislexia do desenvolvimento e as possíveis comorbidades, é escassa a literatura nacional que aborda o tema. Assim, propõe-se um estudo tem como objetivo realizar uma descrição dos resultados obtidos na avaliação multidisciplinar de uma criança com dislexia e verificar a presença de comorbidades associadas ao quadro.

\section{APRESENTAÇÃO DO CASO}

O trabalho foi desenvolvido no Laboratório de Pesquisa em Dificuldades, Distúrbios de Aprendizagem e Transtornos da Atenção (DISAPRE), localizado no Hospital das Clínicas - UNICAMP. O caso selecionado para estudo foi de uma criança do gênero masculino, com 10 anos e 7 meses, cursando $04^{\circ}$ ano (antiga $3^{\text {a }}$ série) do Ensino
Fundamental em uma escola pública da região metropolitana de Campinas-SP e encaminhado pela escola para avaliação e diagnóstico. Inicialmente foi realizada triagem e diante da hipótese diagnóstica de dislexia, foi encaminhado para avaliação específica.

As queixas apresentadas pela escola e pela mãe foram: dificuldades de aprendizagem, principalmente na leitura e escrita; é copista, ainda não sabe ler, pula linhas, é desorganizado, ansioso e triste. Na ocasião da anamnese, a mãe também referiu que a criança apresentava dores pelo corpo e cefaléia.

M.B.F. é filho de um casal não consanguíneo, seus pais são separados e a mãe teve outros dois relacionamentos. No primeiro, teve dois filhos, no segundo (com o pai de M., teve apenas ele) e no último, teve um outro filho que é o caçula.

Mãe realizou pré-natal sem intercorrências médicas. Relatou apenas uma gestação tumultuada. M. foi recém-nascido a termo (idade gestacional de 39 semanas), de parto cesáreo e chorou logo que nasceu. Precisou ficar durante 3 dias na UTI neonatal, pois no parto "foi água para seu pulmão" (segundo informações da mãe) e fez fototerapia, devido a icterícia. Seu peso ao nascer foi de $2,950 \mathrm{~kg}$, estatura de $46 \mathrm{~cm}$ e APGAR de 7 no primeiro minuto e 9 no quinto minuto, Perímetro cefálico de $35,5 \mathrm{~cm}$.

Seu desenvolvimento neuropsicomotor foi dentro dos padrões esperados, de modo que engatinhou com aproximadamente 6 meses e andou sem apoio com 1 ano. No desenvolvimento da fala/linguagem, falou as primeiras palavras aos 2 anos; não formava frases. Falou frases compreensíveis por volta dos 4 anos de idade. Tem sono agitado e mãe referiu parassonias frequentes: sentar, falar, chorar, se mexer enquanto dorme, negou enurese noturna, dorme com a mãe, pois tem medo de dormir sozinho, tem sono diurno.

Quanto ao histórico médico, em 2004, com 7 anos, apresentou dois episódios que foram interpretados como crise epiléptica e iniciou o uso de carbamazepina. Tais episódios ocorriam durante o sono. Esta medicação foi utilizada por dois anos e retirada de uma vez em 2007. Realizou cirurgia de adenoide em 2004.

Com relação à sociabilidade, tem bom relacionamento interpessoal, no entanto, tem poucos amigos e costuma se isolar dos outros. Com relação à escolaridade, M. frequentou o jardim, a pré-escola e entrou na $1^{\text {a }}$ série com 7 anos. Teve três mudanças de escolas, cursando um ano em cada escola. De acordo com a mãe, ele se recusa ir à escola e não "tem vontade de fazer as coisas" (sic). Gosta de matemática e tem bom desempenho nesta matéria. Seu desempenho, nas demais matérias é abaixo da 
média. Faz reforço escolar duas vezes por semana na própria escola em que estuda. Em relação aos antecedentes familiais: pais apresentaram dificuldades escolares.

O processo diagnóstico foi realizado de acordo com a seguinte bateria de testes:

\section{Com a mãe}

a) Roteiro semi-estruturado de anamnese;

b) Inventário de Comportamentos da Infância e Adolescência - $\mathrm{CBCL}^{15}$;

\section{Com a criança}

Avaliação Neuropsicológica:

a) Teste Gestáltico Visomotor Bender - B-SPG ${ }^{16}$;

b) Escala de Inteligência Wechsler para Crianças - WISC-III ${ }^{17}$;

c) Teste Wisconsin de Classificação de Cartas WCST ${ }^{18}$;

d) Teste Luria Nebraska - C ${ }^{19}$;

e) Testes de Cancelamento - TC: Figuras Geométricas e Letras em Fileira ${ }^{20}$;

f) Tower of London - TOL ${ }^{21}$;

g) Trail Making Test - TMT-A/B 22;

h) Stroop Color Word Test - SCWT ${ }^{23}$;

i) Inventário de Depressão Infantil - CDI ${ }^{24}$;

\section{Avaliação Fonoaudiológica}

a) Avaliação Fonológica da Criança ${ }^{25}$;

b) Fala espontânea;

c) Nomeação Automática Rápida - RAN ${ }^{26}$;

d) Prova de Consciência Fonológica - PCF 27;

e) Nível de leitura;

f) Escrita espontânea e sob ditado ${ }^{28}$.

O trabalho foi aprovado pelo Comitê de Ética da Faculdade de Ciências Médicas-UNICAMP sob os protocolos no 215/2007 e 648/2007.

\section{RESULTADOS}

A análise da avaliação neuropsicológica seguiu um Protocolo de Avaliação Neuropsicológica Infantil/PANI-DISAPRE e os resultados dos instrumentos foram organizados em termos das seguintes funções: atenção, memória, funções visuais, auditivas e táteis, funções executivas, funções intelectuais e aspectos afetivo-emocionais. Os aspectos referentes à linguagem foram especificados na avaliação fonoaudiológica. Os resultados das avaliações foram considerados dos pontos de vista quantitativo e qualitativo.

De acordo com os resultados da avaliação neuropsicológica, pôde-se observar que $M$. obteve desempenho intelectual geral no limite inferior da média esperada para sua idade cronológica. Apresentou melhor desempenho em tarefas não-verbais (visuo-espaciais) que em tarefas verbais, com diferença estatisticamente significante $(p<0,01)$ de 16 pontos entre os Quocientes de Inteligência Verbal (QIV) e de Execução (QIE).

$\mathrm{Na}$ Avaliação Atencional, obteve desempenho médio e número de erros não significativos nos testes de cancelamento, porém a análise qualitativa demonstrou uso desorganizado de estratégias de rastreamento visual para a realização das tarefas. Apresentou desempenho médio inferior tanto em tarefas de memória imediata visual quanto em auditiva.

A criança obteve bom desempenho nas funções visuais de discriminação: reconheceu e nomeou adequadamente cores, formas geométricas e objetos; desempenho dentro da média em organização visual e visuo-espacial, porém usou estratégias de ensaio e erro para a resolução das atividades; desempenho no limite inferior da média em atividade que exigia capacidade de planejamento envolvendo eventos sequenciais, causais e temporais. Nas funções auditivas, teve bom desempenho em discriminação, seguir ordens simples, mas dificuldades em reprodução de estruturas rítmicas e compreensão verbal no nível limítrofe da média; bom desempenho em percepção tátil (estereognosia), reconhecendo objetos e texturas.

Quanto à linguagem, demonstrou pouca interação verbal, repertório verbal adequado (vocabulário), dificuldades no estabelecimento de relações entre conceitos (abstração), expressão de conhecimentos gerais e compreensão. Quanto aos aspectos da avaliação de linguagem oral foram observados os seguintes achados: alterações somente no nível semântico, com restrição lexical, sendo que os outros níveis, sintático, fonético, fonológico e pragmático encontram-se de acordo com a idade cronológica.

$\mathrm{Na}$ Avaliação das Funções Executivas obteve bom desempenho em tarefa de conflito cognitivo, que avaliou a capacidade de inibição cognitiva e atenção seletiva visual, não apresentando prejuízo no tempo de reação e no número de erros, indicados pelos escores de interferência. Em tarefa que exigiu capacidade de alternância/flexibilidade cognitiva teve aumento no tempo de reação em função da complexidade da tarefa, sendo que o tempo foi maior no teste que envolveu símbolos linguísticos (letras). Também demonstrou dificuldades na capacidade de modificação de estratégias cognitivas diante das contingências ambientais, não conseguindo completar $01^{\circ}$ contexto do WCST e apresentando maior frequência de escores perseverativos. Também teve dificuldade na auto-percepção 
de erros, monitoramento do próprio desempenho e na capacidade de planejamento com carência de estratégias para auxiliar seu desempenho.

$\mathrm{Na}$ avaliação das Habilidades Escolares foram obtidos os seguintes resultados:

a) Na linguagem escrita tem letra do tipo cursiva, qualidade gráfica média, pinça adequada, velocidade de escrita lenta, presença de sincinesias na boca e língua, nível de escrita silábico (transição para silábico com valor sonoro); realizou cópia de palavras e frases simples. Na escrita espontânea e sob ditado: alterações nas vias de uso da escrita, rotas fonológica e lexical, sendo que não há relação grafema-fonema; início da fase silábica para a escrita. Para a elaboração de texto, há uma restrição lexical, em função desta não utilização da escrita, portanto são elaboradas somente palavras de alta frequência ao seu léxico semântico, o que dificulta a inteligibilidade de sua grafia (Figura 1);

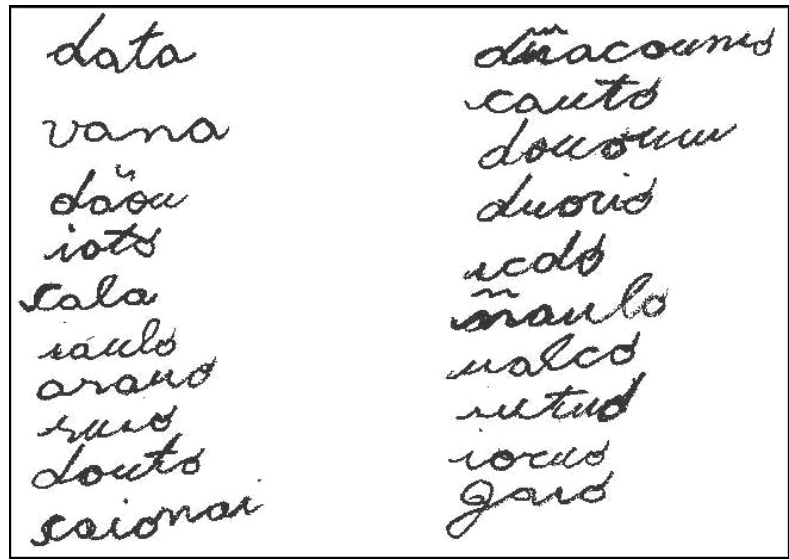

Legenda - coluna da esquerda: data, vela, dado, água, também, vidro, amanhã, palavra, nenhum, tempestade. Coluna da direita: caçador, assaltou, churrasco, queixo, tijolo, comeram, soltou, mangueira, combinar, exemplo.

\section{Figura 1 - Amostra de escrita sob ditado}

b) Na leitura teve dificuldades na discriminação de letras, na leitura de sílabas simples e complexas (encontros consonantais e dígrafos) e, consequentemente, na leitura de palavras simples. Seu nível de leitura é logográfico, em que reconhece os grafemas, porém não é capaz de juntá-los. Na Prova de Nomeação Automática Rápida (RAN) apresentou dificuldades no tempo de nomeação de cores, letras, dígitos e objetos, que exige acesso ao léxico mental, quando comparado ao esperado para sua idade e escolaridade (Figura 2). Na Prova de Consciência Fonológica apresentou alterações em provas fonêmicas e de rima (Figura 3);

c) Cálculo: discriminou algarismos, realizou cálculos simples e solucionou problemas matemáticos oralmente com apoio concreto.

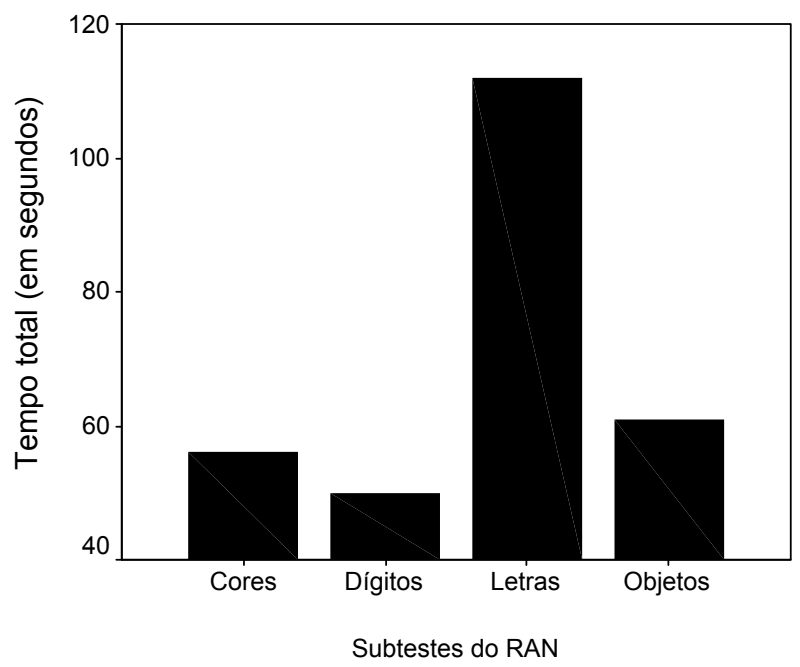

Figura 2 - Tempo de nomeação para cores, letras, dígitos e objetos na Prova de Nomeação automática rápida (RAN)

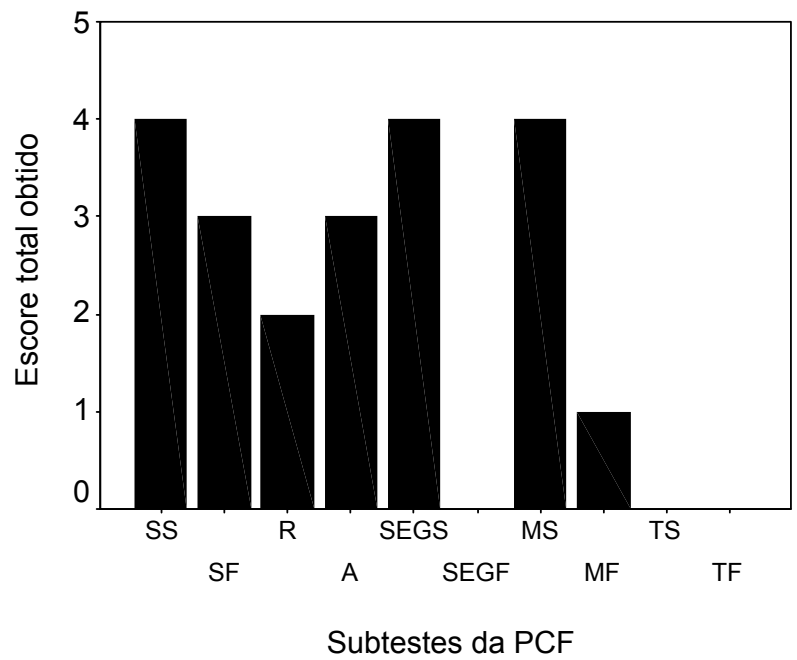

Legenda: SS: síntese silábica, SF: síntese fonêmica, R: Rima, A: aliteração, SEGS: segmentação silábica, SEGF: segmentação fonêmica, MS: manipulação silábica, MF: Manipulação fonêmica, TS: transposição silábica, TF: transposição fonêmica

Figura 3 - Número de acertos na Prova de Consciência Fonológica (PCF) 


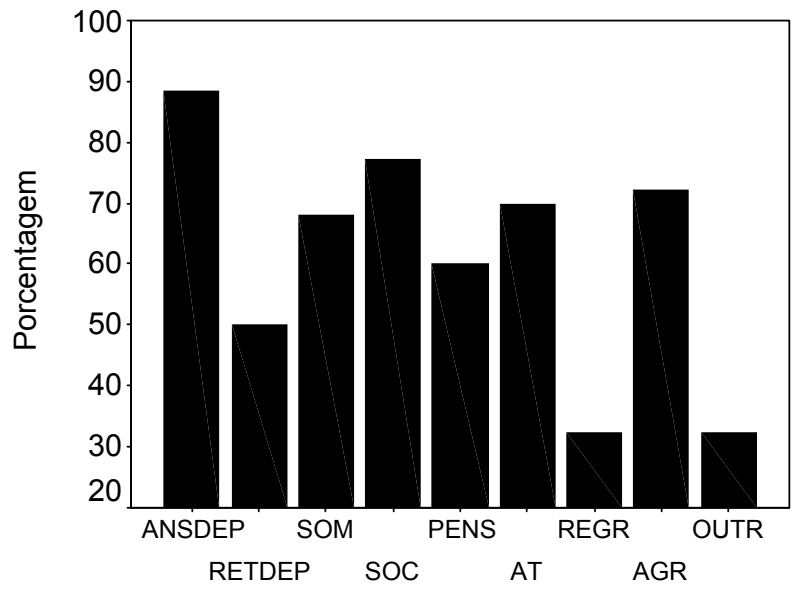

Subtestes do CBCL

Legenda: Ans/Dep: Ansiedade/Depressão; Retr/Dep: Retraimento/Depressão; Som: Somáticas; Soc: Sociais; Pens: Pensamento; At: Atenção; Regr: Desrespeito às regras; Agr: Agressividade; Outr: Outras.

Figura 4 - Porcentagem de queixas familiares no teste CBCL

Durante as avaliações foi observado que $\mathrm{M}$. queria desistir da tarefa quando não conseguir realizá-la e necessitava de auxílio e maior incentivo para responder às questões feitas em algumas atividades. Na avaliação de sintomas depressivos, por meio do auto-relato com o CDI, não ultrapassou o ponto de corte, no entanto, houve relatos de temor com relação aos acontecimentos futuros, preocupação com sua aparência, pouca diversão no contexto escolar, preocupação e comparação de seu desempenho com o de seus pares.

De acordo com o relato familiar de queixas comportamentais e emocionais (Figura 4), houve porcentagem maior nos escores de queixas relacionadas à: depressão/ansiedade, relacionamento social, comportamentos agressivos, problemas de atenção, queixas somáticas e problemas no pensamento.

As dificuldades apresentadas por M. na escola repercutiram nos aspectos emocionais e comportamentais, de modo que ele passou a apresentar: avaliação negativa de seu desempenho, generalização de baixa auto-eficácia para outras esferas de sua vida, formação de auto-conceito negativo, comportamento apático, ausência de prazer (anedonia), sentimentos de tristeza e recusa em ir à escola.

Os resultados do processo avaliativo foram compatíveis com Transtorno Específico de Leitura (F 81.0 - Dislexia do Desenvolvimento - CID-10) como quadro primário, tendo como comorbidade o quadro de depressão infantil. Para o diagnóstico da dislexia foram excluídas outras possíveis causas para as dificuldades: rebaixamento intelectual, déficit sensorial, motor, sociocultural, problemas psicológicos. Para o quadro depressivo, a avaliação clínica e com a mãe, indicou que a criança preencheu os critérios da CID-10 para episódio depressivo, assim como os critérios indicados por Poznanski, Mokros e Grossman ${ }^{29}$, específicos para depressão infantil: A - Humor, conduta ou aparência depressiva; B - Pelo menos 4 ou 5 dos seguintes sintomas: retraimento social, problemas de sono, queixas ou fadiga, hipoatividade, anedonia, baixa auto estima, dificuldade no trabalho escolar, ideação mórbida ou suicida; C - duração mínima de 1 mês.

A conduta interdisciplinar se deu por meio do encaminhamento para intervenção fonoaudiológica, com objetivo da maximização das habilidades de leitura e escrita, psicológica e psiquiátrica para acompanhamento da comorbidade depressiva.

\section{DISCUSSÃO}

Com a avaliação neuropsicológica foi possível evidenciar uma das principais discrepâncias e critérios de exclusão observados nos indivíduos com distúrbios de aprendizagem que é a dificuldade nas habilidades escolares apesar do adequado nível intelectual. Além disso, foi indicada a relação entre os déficits em funções corticais superiores e o desempenho escolar.

Observou-se que M. apresentou nível intelectual de acordo com a expectativa para crianças de sua faixa etária, com melhor desempenho em tarefas visuo-espaciais mediadas pelo hemisfério cerebral direito. Os resultados também demonstraram bom desempenho em funções das áreas primárias e mais dificuldades em áreas de associação secundárias e terciárias.

Também foram observadas dificuldades nas funções atencionais e executivas. Há uma associação próxima entre as habilidades de leitura e escrita e a ativação do lobo frontal e os estudos têm descrito déficits em certos aspectos das funções executivas em crianças com dislexia ${ }^{10}$. Com relação a este aspecto, M. teve desempenho prejudicado em planejamento, flexibilidade cognitiva, resolução de problemas, no uso de estratégias cognitivas e sua modificação, na auto-percepção de erros e monitoramento do próprio desempenho. Tais achados sugerem possíveis problemas com o recrutamento de recursos cognitivos para a resolução de tarefas mais complexas e que requerem maior integração e controle dos estímulos ambientais.

Desse modo, verifica-se que no processo de leitura é necessária a integração das informações provenientes do processamento visual (discriminação, 
organização visual e visuo-espacial) dos símbolos gráficos (grafemas/letras), processamento auditivo/ linguístico (decodificação fonológica, conversão grafema-fonema) ${ }^{30,31}$ e mediação pelo controle atencional e funções executivas.

As consequências do quadro da dislexia são diversas, uma vez que compromete não só os aspectos pessoais da criança (neuropsicológico e emocional), mas também tem repercussões na família, escola e no relacionamento interpessoal. No caso estudado, a dislexia foi acompanhada por alterações nos aspectos afetivo/emocionais, uma vez que a criança desenvolveu um quadro depressivo, corroborando com outros estudos indicados na literatura ${ }^{12,32}$. O fato de a criança apresentar um quadro de distúrbio de aprendizagem pode ser considerado um fator de risco para o desenvolvimento de transtornos psicológicos.

A avaliação da sintomatologia depressiva em crianças possui alguns obstáculos, dentre eles a dificuldade da criança discriminar, reconhecer e nomear seus sentimentos. $\mathrm{Na}$ avaliação realizada por meio do auto-relato com o CDI este aspecto ficou evidente, uma vez que M. não ultrapassou o ponto de corte para a sintomatologia significativa, dado que não corroborou a avaliação clínica e com a família.

A presença da depressão associada à dislexia também pode contribuir para o aumento da extensão dos déficits neuropsicológicos, já que a sintomatologia depressiva está associada ao rebaixamento na capacidade atencional, prejudicando o desempenho escolar ${ }^{14}$.

\section{CONCLUSÃO}

A dislexia é um quadro com etiologia multifatorial e que compromete diversas funções neuropsicológicas (dentre elas, a atenção e as funções executivas) e o distúrbio de aprendizagem pode ser considerado um fator de risco para o desenvolvimento de transtornos psicológicos.

Diante disso, fica clara a importância da avaliação criteriosa, de modo a identificar a extensão e especificidades dos déficits e promover uma interlocução dos resultados de diferentes áreas do conhecimento para auxiliar na precisão diagnóstica e planejamento da intervenção.

\begin{abstract}
Purpose: to analyze features in a case of dyslexia with childhood depression. Method: description of evaluation's results in a disciplinary case with dyslexia and depression. This study comprised a child, masculine gender, with 10 years and 7 months, studying the $4^{\text {th }}$ year of the Elementary Teaching in a public school. Results: the results of this study evidenced alterations not only in language, where there are difficulties in the phonological information processing, as well as in attention and executive functions and comorbidity of childhood depression. Conclusion: the study revealed the need for evaluation by an interdisciplinary team for a precise diagnosis and, consequently a better intervention in children with dyslexia and their co-morbidities.
\end{abstract}

KEYWORDS: Dyslexia; Evaluation; Depression; Comorbidity

\section{REFERÊNCIAS}

1. DSM-IV-TR. Manual diagnóstico e estatístico de transtornos mentais. 4. ed. Porto Alegre: Artes Médicas; 2002.

2. Artigas-Pallarés J. Dislexia: enfermedad, transtorno o algo distinto. Rev Neurol 2009; 42(Supl 2):S63-9.

3. Boder E. Developmental dyslexia: a diagnostic approach based on three atypical reading-spelling patterns. Dev Med Child Neurol. 1973; 15(5):663-87.

4. Torppa M, Tolvanen A, Poikkeus AM, Eklund K, Lerkkanen MK, Leskinen E, et al. Reading development subtypes and their early characteristics. Ann Dyslexia. 2007; 57:3-32.

5. Pino JAH, Harb SL, Bassi NSJ, Samper GPS. Fundamentos neuropsicológicos de la dislexia evolutiva. Psicología desde el Caribe. 2007; 19: 222-68.

6. Moores E. Deficits in dyslexia: barking up the wrong tree? Dyslexia. 2004; 10:289-98.

7. Shaywitz SE, Shaywitz BA. Dyslexia (Specific reading Disability). Biol Psychiatry 2005;57:1301-9.

8. Salgado SA, Pinheiro A, Sassi AG, Tabaquim MLM, Ciasca SM, Capellini SA. Avaliação fonoaudiológica e neuropsicológica na dislexia 
do desenvolvimento do tipo mista: relato de caso. Salusvita. 2006; 25(1):91-103.

9. Valdois S, Bosse ML, Tainturier MJ. The cognitive deficits responsible for developmental dyslexia: review of evidence for a selective visual attentional disorder. Dyslexia. 2004; 10:339-63.

10. Reiter A, Tucha O, Lange KW. Executive functions in children with dyslexia. Dyslexia. 2005; 11:116-31.

11. Maag JW, Reid R. Depression among students with learning disabilities: assessing the risk. J Learn Disabil. 2006; 39(1):3-10.

12. Arnold EM, Goldston DB, Walsh AK, Reboussin BA, Daniel SS, Hickman E, Wood FB. Severity of emotional and behavioral problems among poor and typical readers. J Abnorm Child Psychol. 2005; 33(2):205-17.

13. Maughan B, Rowe R, Loeber R, StouthamerLoeber M. Reading problems and depressed mood. J Abnorm Child Psychol. 2005; 31(2):219-29.

14. Lima RF, Massoni I, Ciasca SM. Depression symptons and neuropsychological functions in children with learning difficulties. 13th International European Society for Child and Adolescent Psychiatry Congress. Florence/ltaly; 2007.

15. Bordin IAS, Mari JJ, Caeiro MF. Validação da versão brasileira do "Child Behavior Checklist" (CBCL) (Inventário de comportamentos da infância e adolescência): dados preliminares. Rev ABPAPAL. 1995; 17:55-66.

16. Sisto FF, Noronha APP, Santos AAA dos. Teste Gestáltico Visomotor de Bender - Sistema de Pontuação Gradual (B-SPG). Manual. São Paulo: Vetor; 2005.

17. Figueiredo VLM. WISC-III: Escala de Inteligência Wechsler para crianças - manual. Adaptação e padronização de uma amostra brasileira. São Paulo: Casa do Psicólogo; 2002.

18. Cunha JAC, Trentini CM, Argimon IL, Oliveira MS, Werlang BG, Prieb RG. Teste Wisconsin de Classificação de Cartas: Manual revisado e ampliado. São Paulo: Casa do Psicólogo; 2005.

19. Lima RF, Mello RJL, Massoni I, Riechi TIJS, Ciasca SM. Teste Luria Nebraska-C. Departamento de Neurologia. FCM - UNICAMP; 2005 (não publicado).

http://dx.doi.org/10.1590/S1516-18462010005000055

RECEBIDO EM: 05/07/2009

ACEITO EM: 06/02/2010

Endereço para correspondência:

Ricardo Franco de Lima

Rua Jorge Miranda, 104 ap. 43

Campinas - SP

CEP: $13020-180$

E-mail: rilima@fcm.unicamp.br
20. Lima RF, Ciasca SM. Testes de Cancelamento (TC): adaptação DISAPRE. Universidade Estadual de Campinas. Faculdade de Ciências Médicas Departamento de Neurologia: Campinas/SP; 2006 (não publicado).

21. Lima RF, Ciasca SM. Teste Torre de Londres (TOL): adaptação DISAPRE. Universidade Estadual de Campinas. Faculdade de Ciências Médicas Departamento de Neurologia: Campinas/SP; 2007 (não publicado).

22. Lima RF, Ciasca SM. Teste das Trilhas A/B (TMT - A/B): adaptação DISAPRE. Universidade Estadual de Campinas. Faculdade de Ciências Médicas - Departamento de Neurologia: Campinas/ SP; 2006 (não publicado).

23. Lima RF, Ciasca SM. Teste Cor-Palavra de Stroop (SCWT): adaptação DISAPRE. Universidade Estadual de Campinas. Faculdade de Ciências Médicas - Departamento de Neurologia: Campinas/ SP; 2006 (não publicado).

24. Gouveia VV, Barbosa GA, Almeida HJF, Gaião AA. Inventário de depressão infantil - CDI: Estudo de adaptação com escolares de João Pessoa. J Bras Psiquiatr. 1995; 44:345-9.

25. Yavas M, Hernandorena CLM, Lamprecht RR. Avaliação fonológica da criança- reeducação e terapia. Porto Alegre: Artes Médicas; 1991.

26. Ferreira TL, Capellini AS, Ciasca SM, Tonelotto JMF. Desempenho de escolares leitores proficientes na prova de velocidade de recodificação fonológica. Temas Desenvolv. 2003; 12(29):26-32.

27. Capovilla AGS, Capovilla FC. Prova de consciência fonológica: desenvolvimento de dez habilidades da pré-escola à segunda série. Temas Desenvolv. 1998; 7(37):14-20.

28. Pinheiro AMV. Leitura e escrita: uma abordagem cognitiva. Campinas: Psy II; 1994.

29. Poznanski E, Mokros HB, Grossman J. Diagnostic criteria in childhood depression. Am J Psychiatr. 1985; 142(19):1168.

30. Capellini SA, Ferreira TL, Salgado CA, Ciasca SM. Desempenho de escolares bons leitores, com dislexia e com transtorno do déficit de atenção em nomeação automática rápida. Rev Soc Bras Fonoaudiol. 2007; 12(2):114-9.

31. Salgado CA, Capellini SA. Phonological remediation program in students with developmental dyslexia.Pró-Fono Revista de Atualização Científica. 2008 jan-mar;20(1):31-6.

32. Salgado CA, Lima RF, Ciasca SM. Neuropsychological and phonoaudiological findings in developmental dyslexic children at the Hospital das Clínicas - Unicamp/Brazil. Meeting International Neuropsychological Society; Buenos Aires, Argentina; 2008. 